\title{
EVALUACIÓN DE UN PROCESO CONTÍNUO DE BURBUJEO PARA RECUPERACIÓN DE ALMIDÓN Y MUCILAGO DE ÑAME.
}

\section{EVALUATION OF A CONTINUOUS PROCESS OF BUBBLING FOR RECOVERING YAM STARCH AND MUCILAGE.}

\author{
Jhonys J. Pérez ${ }^{1}$, Alfredo C. Fernández² y Jairo G. Salcedo ${ }^{3}$. \\ Recibido para publicación: Octubre 15 de 2014 - Aceptado para publicación: Mayo 4 de 2015
}

\begin{abstract}
RESUMEN
La extracción de almidón de tubérculos de ñame se dificulta por la presencia de un material gelatinoso (mucílago), que obstaculiza la sedimentación del almidón y altera las características del mismo. Por lo cual, el presente trabajo tuvo como objetivo evaluar un proceso de burbujeo continuo, por inyección de aire, para extraer y recuperar almidón y mucílago de $D$. alata y $D$. rotundata. Cuatro relaciones ñame/agua $(1 / 4,1 / 5,1 / 6$ y $1 / 7)$ p/p y tres períodos de operación (30,60 y 90min) fueron usados bajo un diseño experimental factorial. Se determinó que el proceso de extracción de almidón y mucílago es dependiente de la relación ñame - agua y tiempo del período de operación, existiendo diferencias significativas en las operaciones en las cuales se empleó un tiempo de 60 minutos y se adicionó $6 \mathrm{~L}$ de agua por kilogramo de ñame. Tambien se observó, que la mayor extracción de almidón se presentó en $D$. rotundata, mientras que la mayor cantidad se mucílado se extrajo en $D$. alata.
\end{abstract}

Palabras Clave: Almidón, ñame, mucilago, burbujeo.

\begin{abstract}
The extraction of starch from yam tubers is difficult because of the presence of a gelatinous substance (mucilage), that makes hard to separate starch from tubers and also change its quality its characteristics. The objective of this study was to evaluate a process of continuous bubbling to extract and recover starch and mucilage from D. alata and D. rotundata. Four rations of $\mathrm{kg}$ of yam tuber / water (1/4, 1/5, $1 / 6$ and 1/7) w/ w and three operation period (30,60 and $90 \mathrm{~min}$ ) were used in a factorial experimental design. The extraction process to separate mucilage and starch from yam tubers was influenced by the ratio of $\mathrm{kg}$ of yam tuber/water and time with significant differences in operations in which they used a time of 60 minutes and $6 \mathrm{~L}$ of water per kilogram of yam was added;.Starch yield higher on D. rotundata, while the higher yield on mucilage was in D. alata.
\end{abstract}

Key-words: starch, yam, mucilage, bubbling.

1'Ingeniero Agrícola; Joven investigador COLCIENCIAS; e-mail: jhjperezbo@unal.edu.co; dirección: Cll 42G \#17-02

${ }^{2}$ Magister en Ciencia y Tecnología de Alimentos; Docente Universidad de Sucre, Colombia, e-mail:Alcaferqui2004@yahoo.es; dirección: Cra 12C № 23-126

${ }_{3}^{3}$ ph.D Ingeniería Química; Docente Universidad de Sucre, Colombia, e-mail: Jairo.salcedo@unisucre.edu.co, direccion Cll 23A N²5-114 


\section{INTRODUCCIÓN}

El ñame (Dioscorea spp.), es uno de los seis géneros pertenecientes a la familia de las Dioscoraceae y agrupa aproximadamente 600 especies, de las cuales solamente 12 son comestibles, se presenta como una enredadera y se caracteriza por la presencia de tubérculos subterráneos y/o aéreos (bulbillos); los cuales son ricos en almidón y mucílago (Coursey 1980).

El ñame es un cultivo de importancia socioeconómica, principalmente en los países en desarrollo del trópico, donde es de amplio consumo por la población rural. Según FAOSTAT (2013), para el año 2012 se produjeron a nivel mundial alrededor de 57 millones de toneladas de este tubérculo, de las cuales el continente africano aporta el 96\%. En Colombia, el cultivo de este vegetal es de gran trascendencia en la costa norte del país (Agronet 2013), donde se concentran la gran mayoría de tierras agrícolas dedicadas a este cultivo, principalmente de las especies Dioscorea alata (ñame criollo) y Dioscorea rotundata (ñame espino) (Reina 2012).

Su condición de alimento regional que no se sitúa como de primera necesidad, ha estancado su explotación, la cual se realiza generalmente en predios de economía campesina con bajo nivel de tecnificación, debido a que no cuentan con alternativas tecnológicas de transformación direccionadas a dar valor agregado bajo esquemas de producción agroindustriales (Hata et al. 2003).

El ñame es un alimento rico en proteínas, calorías y almidón que lo hace semejante al maíz, tanto en sabor, como en textura y color; es empleado por industrias alimenticias con la misma finalidad que el almidón de maíz (ANUÁRIO 1994), asi mismo, es reconocido en el ñame la presencia de un tipo de sustancia gomosa; denominada como, mucilago, la cual, desde el punto de vista físico es un sistema coloidal tipo gel; constituido por agua, pectinas, azúcares y ácidos orgánicos (Fu et al. 2014).

El mucílago de ñame le confiere un sin número de propiedades funcionales, tanto en la industria alimenticia como en la farmacéutica; su viscosidad y propiedades emulsificantes posibilitan el uso de este en diferentes productos (Aparecida et al. 2011), debido a que el mucílago viscoso de este tubérculo se compone principalmente de diosgenina precursor de progesterona, cortisona y otros esteroides de amplia utilización farmacéutica (Tsaj y Tai 1984).

Por otro lado, la extracción de almidón de yuca es sencilla, a diferencia de otros tubérculos que contienen almidón como el ñame debido a que la solución de los gránulos de almidón, a menudo es obstaculizada por la presencia de varios componentes como el mucílago. Esto interfiere en el proceso de molienda y conduce no sólo a la pérdida de almidón, sino también a una disminución de la calidad del almidón extraído (Fu et al. 2014).

Debido a esto, en el presente estudio se plantea la evaluación de un proceso continuo de burbujeo para la separación y recuperación de almidón y mucílago de dos especies de ñame (D. rotundata y D. alata), sin el uso de aditivos químicos indeseables en el proceso. 


\section{MATERIALES Y MÉTODOS}

Los tubérculos de ñame (D. alata y $D$. rotundata) fueron recolectados en predios de agricultores locales en el departamento de Sucre (Colombia), en el mes de agosto de 2012. El estudio se realizó en las instalaciones de la unidad de procesos unitarios de la universidad de Sucre (Latitud 9 $9^{\circ} 11^{\prime} 40.15^{\prime \prime}$ $\mathrm{N}$ y longitud $75^{\circ} 24^{\prime} 10.77^{\prime \prime} \mathrm{O}$ ). El equipo de burbujeo continúo fue desarrollado por Pérez y Sayas (2010).

La materia prima fue obtenida (ñame) de los productores locales, los cuales, se cosecharon a los 9 meses de plantado, posteriormente se realizó un acondicionamiento que consistió en limpieza, pelado y trozado (3 cm de espesor), para la posterior elaboración de la pasta de ñame, por medio del licuado de los trozos de ñame, con una licuadora de uso doméstico.

El agua empleada para este fin se adicionó teniendo en cuenta las relaciones $\mathrm{p} / \mathrm{p}$ ñame / agua evaluadas $(1 / 4,1 / 5,1 / 6$ y $1 / 7)$, con base en relaciones empleadas en estudios realizados por Fu et al. (2005).

Seguido a esto y mediante el empleo de un equipo de bombeo (centrifugo de 0,25 hp) incorporado en el dispositivo de burbujeo continuo, se hizo pasar la pasta a la torre I hasta alcanzar la mitad de su altura; adicionalmente se inyecto aire comprimido proveniente de un compresor (Afm de 100 libras), el cual fue distribuido mediante un plato poroso de $12 \mathrm{~cm}$ de diámetro (diámetro de poro de 0,5 mm), con el propósito de generar burbujas de aire que posibiliten la separación del mucílago o material gelatinoso de las partículas de almidón.
En el funcionamiento del equipo, el fluido espumoso generado en la torre I, se moviliza hacia las torres sub-siguientes.

La torre II realiza un refinamiento o control más estricto de la mezcla proveniente de la torre I, mediante el uso de aire comprimido, el cual es distribuido mediante un plato poroso con orificios de $0,5 \mathrm{~mm}$.

En la torre III se espera que el mayor porcentaje de fluido espumoso, material gelatinoso (mucílago), concentrado en la periferia de las burbujas formadas previamente, las que Ilegan a esta torre y son destruidas mediante el uso de alcohol etílico al 70\%.

Este procedimiento, desde la torre I hasta la III fue estudiado por periodos de 30, 60 y $90 \mathrm{~min}$. Posteriormente esta mezcla se destiló para obtener el mucílago y recuperar el alcohol etílico.

La extracción del almidón, inició con la colecta de la pasta baja en mucilago, sedimentada en la parte inferior de las torres I y II, la cual sé filtro con tela nylon, para retirar la fibra de la pasta lechosa. Consecutivamente, este material filtrado se dejó en sedimentación por un periodo de 8 horas y se le retiro el sobrenadante presente.

Luego el almidón húmedo fue sometido a secado por 24 horas a temperatura ambiente (promedio de $28^{\circ} \mathrm{C}$ ), para después ser secado en horno a temperatura de $40{ }^{\circ} \mathrm{C}$, hasta peso constante por 72 horas. 
El porcentaje de almidón extraído, se ha expresado como la relación entre el peso del almidón seco y el peso fresco de ñame usado en cada ensayo (Daiuto et al. 2005).

Por otro lado, el porcentaje de mucílago, se registró a partir de la destilación de la mezcla contenida al final de cada periodo en la columna III, cuyo producto se pesó al final de este proceso y relacionado con el peso fresco de ñame correspondiente.

Para el análisis estadístico de los datos fue empleado un diseño factorial $2 \times 3 \times 4$, bajo un esquema de aleatorización completamente al azar, donde el factor especie asumió dos componentes $(D$. alata y $D$. rotundata), el factor tiempo tuvo tres niveles y el factor relación ñame / agua cuatro relaciones; con tres repeticiones por combinación tiempo - relación ñame / agua, para un total de 36 corridas experimentales por especie de ñame. Este procedimiento fue realizado con el uso de software estadístico SAS ${ }^{\circledR}$ versión 9.1. Para la comparación de medias se utilizó la prueba de Tukey con un nivel de significancia del $5 \%$.

\section{RESULTADOS Y DISCUSIÓN}

En términos generales, el equipo de burbujeo extrajo en promedio $11,70 \%$ de almidón con base al peso de tubérculos frescos evaluadas, con un coeficiente de variación del 68,14\%. El cual, difiere de lo encontrado por Corpoica (2003), donde se obtuvieron valores promedios de extracción de almidón de ñame (variedad Criollo y Espino), cercanos al 25,5\%, consumiendo en el proceso empleado para este fin, aproximadamente
36,8 L/tubérculo, sistemas automáticos de tamices, ralladoras mecánicas y centrifugas; mientras que en el presente estudio se tuvo un gasto máximo de $7 \mathrm{~L}$ de agua por $\mathrm{kg}$ de ñame. Así mismo, se logró recuperar en promedio el 1,50\% del mucilago con base en peso fresco del tubérculo en las especies de ñame evaluadas, con un coeficiente de variación del 68,05\%.

Del análisis de varianza de los datos, se obtuvo que los factores: especie, tiempo y relación ñame/agua, mostraron tener interacción significativa ( $p<0.05)$, es decir que, la cantidad de almidón extraído y/o mucilago recuperado es influenciada por cualquiera de las combinaciones entre los niveles de dichos factores.

\section{Rendimientos de almidón}

Los rendimientos en extracción de almidón por burbujeo en $D$. alata, mostraron diferencias significativas $(p<0.05 \%$ ) en las relaciones ñame/agua evaluadas (Figura 1), en las cuales, es destaca la relación 1/4, encontrándo en esta los niveles más bajos en cuanto a rendimiento, mientras que en las demás relaciones evaluadas el comportamiento de extracción de almidón no se obtuvo diferencias significativas; contrario a los estudios realizados por Daiuto et al. (2005), en el que obtuvo rendimientos en extracción de almidón en $D$. alata al redor del 19 \% en base húmeda, usando tres agentes químicos (pectinasa, hidróxido de sodio y acido oxálico/ oxalato de amonio) y agua como métodos de extracción, con relaciones ñame/agua de 1/2, bajo cámaras de enfriamiento a $2{ }^{\circ} \mathrm{C}$.

De acuerdo a Alvis et al. (2008), encontraron en la caracterización de variedades $D$. alata, 


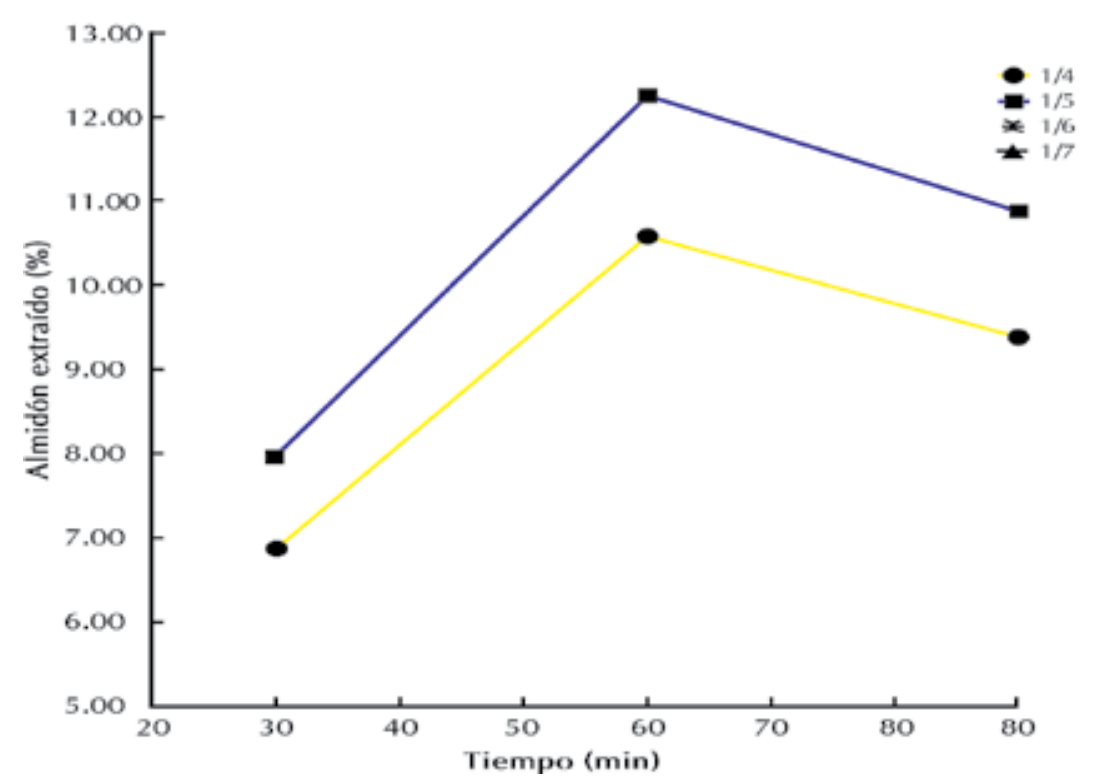

Figura 1. Extracción de almidón (D. alata) en diversos tiempos de operación del equipo de burbujeo y diferentes relaciones ñame/agua

que el contenido promedio de almidón extraído mediante centrifugación como método de remoción del mucilago fueron superiores al 26\%; el cual, está por encima del encontrado en la presente investigación, en donde se alcanza un máximo de extracción del $12 \%$, por lo cual, debe considerase la relación costo beneficio en ambos procedimientos.

Por otra parte, los bajos rendimientos de almidón, encontrados en las mínimas adiciones de agua, se relacionan estrechamente con la alta viscosidad de la pasta de ñame, debida principalmente a la presencia del mucilago, el cual impide la adecuada sedimentación de las partículas de almidón (Fu et al. 2005).

De igual forma, al comparar los tiempos de operación del equipo, se encontró que para todos los casos, el periodo de $30 \mathrm{~min}$ tuvo tendencia a expresar bajos niveles de extracción de almidón, mientras que la operación del equipo de burbujeo por
60 min, es la más adecuada en lo referido a rendimiento de almidón; en cambio, el tiempo de 90 min disminuye el rendimiento de almidón en el equipo.

Esto probablemente puede ser explicado por el alcance del equipo, puesto que el tiempo necesario para aislar de la pasta el mayor porcentaje de mucilago es de $60 \mathrm{~min}$ de operación, posterior al cual, la recirculación en las torres I y II, tiende no ser eficiente en la obtención de almidón, lo cual, se asume es debido a la recirculación de la pasta en el equipo, donde puede estar enriqueciéndose en mucilago, con el volumen estático del tanque principal de alimentación del equipo.

De la misma manera, en D. rotundata

(Figura 2), se mantiene la tendencia mostrada por el proceso, en la cual la relación ñame/ agua de menos rendimiento en almidón es $1 / 4$ y el mejor tiempo para el proceso lo presenta el tiempo de $60 \mathrm{~min}$. 


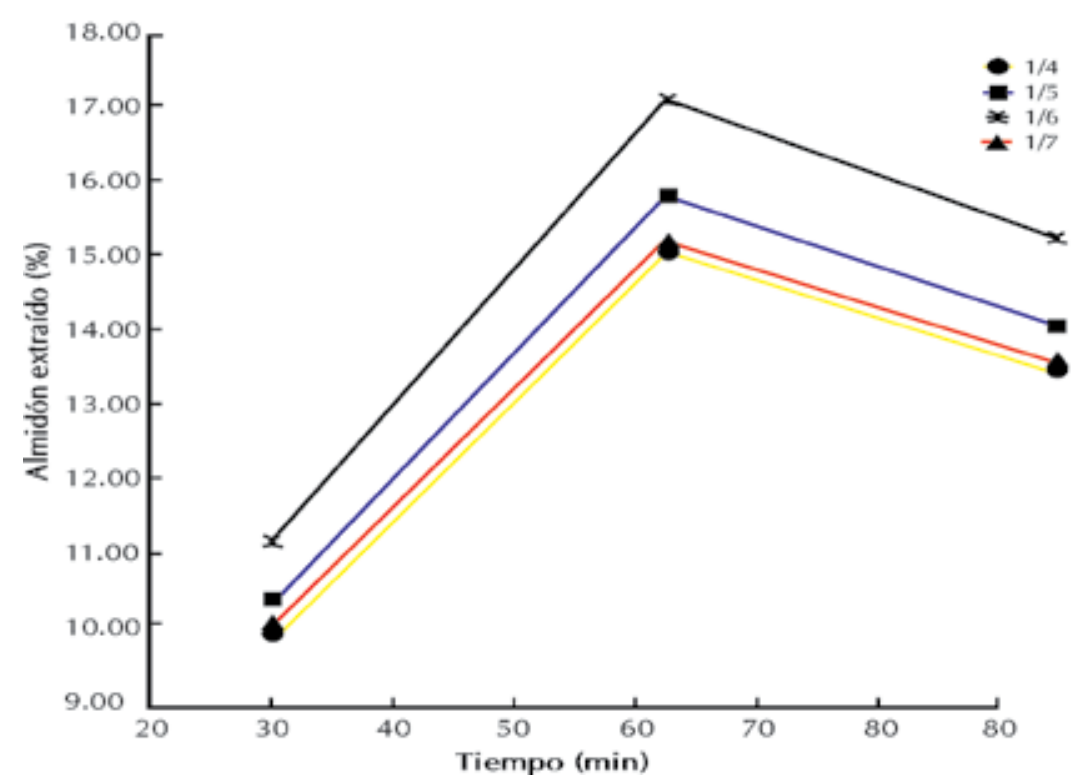

Figura 2. Extracción de almidón (D. Rotundata) en diversos tiempos de operación del equipo de burbujeo y diferentes relaciones ñame/agua.

En el caso del ñame espino, se observa la influencia de la cantidad de agua en el proceso, haciendo notorio que para el caso de la relación $1 / 6$, se obtienen los mejores rendimientos $(16,5 \%)$, a demás; no es muy clara la diferencia entre aplicar 4 y 7 litros de agua por kilo de ñame.

En términos generales, los rendimientos de almidón variaron entre 14\% y 16,5\% (Figura 3), con valores superiores a los reportados por Salcedo et al. (2010), en donde se extrajo almidón de ñame de la variedad espino, por medio del uso de amoníaco $(0,03 \mathrm{M})$, como agente de precipitación de mucílagos, con rendimientos de almidón del 13,7\% para dicha variedad.

De acuerdo a Vidal (2010), en la elaboración de jarabes edulcorantes se encontró un rendimiento en la extracción de almidón de ñame (D. rotundata) de $22,8 \%$, usando un método convencional similar al de la extracción de almidón de yuca y una relación de $10 \mathrm{~L}$ de agua por kg de ñame; está extracción representa un valor superior al encontrado para ñame espino en el equipo de burbujeo, donde se observó un máximo de extracción de almidón de $17 \%$ con una adición de agua de $6 \mathrm{~L}$ por kilogramo de ñame en tan solo 60 minutos.

Por otro lado, es destacable en D. rotundata, que con 60 min de operación del equipo y una relación ñame/agua de $1 / 7$, puede alcanzarse el mismo valor que se alcanzaría con $90 \mathrm{~min}$ del proceso y adicionando 6 litros de agua por kilogramo de ñame fresco, es decir, se estaría disminuyendo el tiempo de operación, pero se traduce en gasto de agua.

En síntesis, se observa en la figura 3, la influencia que tiene la especie de ñame (D. rotundata, D. alata) en la extracción de almidón, en la cual puede apreciarse que la especie $D$. rotundata muestra mejores resultados para la extracción de almidón, cuya diferencia es debida a aspectos genéticos de acumulación de almidón entre especies (Corpoica 2003). 


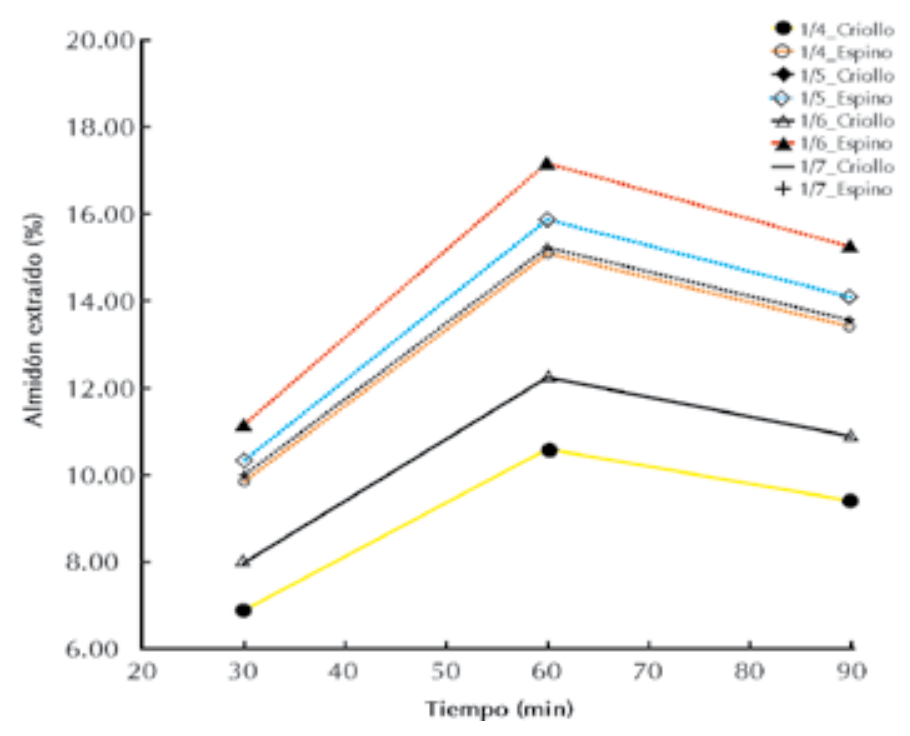

Figura 3. Extracción de almidón en diversos tiempos de operación del equipo de burbujeo y diferentes relaciones ñame/agua para ñame espino y criollo.

Sin embargo, en ambas especies, la extracción de almidón por burbujeo es influenciada por el tiempo dedicado a dicho proceso, el cual, mostró los mejores resultados a los 60 min de operación el tiempo y adición de $6 \mathrm{~L}$ de agua por kilogramo de ñame.

\section{Recuperación de mucílago}

La recuperación de mucílago en el proceso de burbujeo muestra ser sensible a los niveles de agua en el proceso y al tiempo de duración del mismo. La figura 4, muestra como en el ñame criollo, es más eficiente la recuperación de mucílago para la relación ñame/agua 1/7, encontrándo diferencia significativa entre todas las relaciones con $\mathrm{p}<0.05$.

Con respecto al tiempo de operación del proceso, es posible afirmar que existe en este caso una tendencia generalizada en todas las relaciones probadas a presentar los mayores rendimientos en el tiempo de 90 min de operación.
Así mismo, los niveles de recuperación de mucilago estuvieron alrededor del $1,5 \%$ del peso fresco de ñame, por debajo a los reportados por Yen et al. (2008), en un estudio de la influencia del contenido de agua y mucílago en las características fisicoquímicas del almidón de D. alata, encontrando un porcentaje de contenido de mucílago del 4,9\%.

En el ñame espino las recuperaciones de mucílago, por el contrario fueron de alrededor $1,4 \%$, como se observa la figura 5 . Las relaciones agua ñame marcan un efecto diferenciado en la recuperación de mucílago, en los cuales la relación 1/6 presenta los mejores aportes en recuperación.

Del mismo modo adicionar 4 litros de agua por kilogramo de ñame, representa los más bajos rendimiento de mucilago. El tiempo de mejor recuperación el proceso es de $90 \mathrm{~min}$, así mismo el tiempo posee tendencia a aumentar los rendimientos de mucilago cuando este se va incrementándo sin marcar un límite de descenso de recuperación en mucílago. 
TEMAS AGRARIOS - Vol. 20:(1) Enero - Junio 2015 (71 - 80)

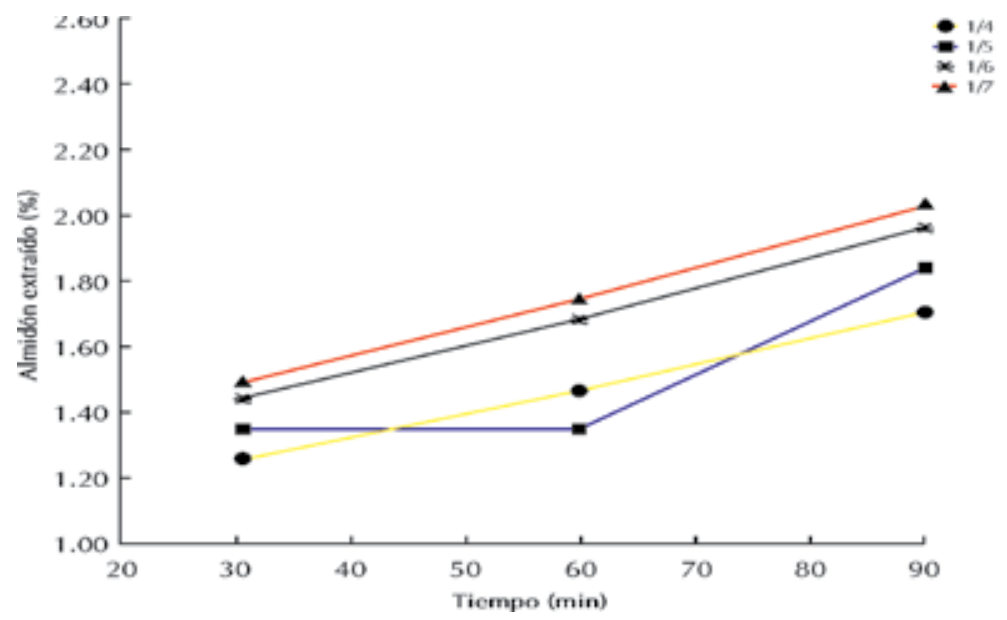

Figura 4. Extracción de mucilago (D. alata) en diversos tiempos de operación del equipo de burbujeo y diferentes relaciones ñame/agua.

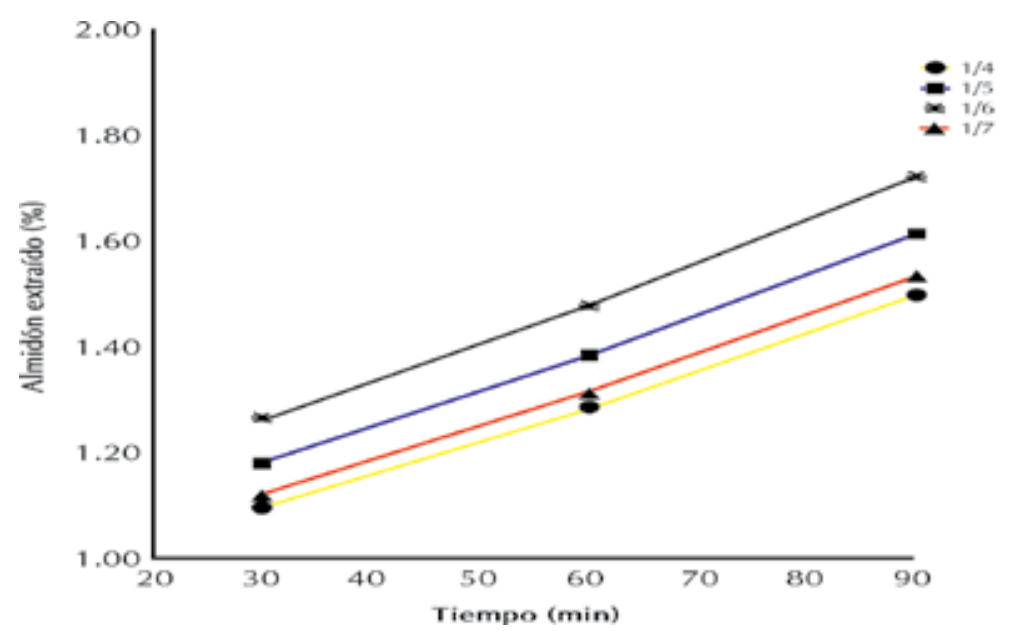

Figura 5. Extracción de mucilago (D. Rotundata) en diversos tiempos de operación del equipo de burbujeo y diferentes relaciones ñame/agua.

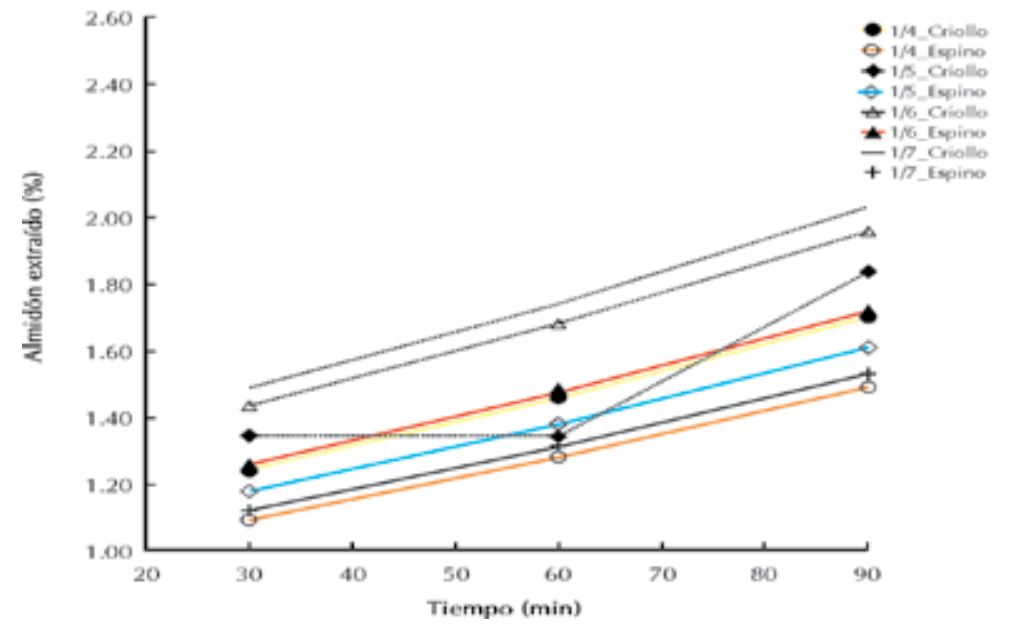

Figura 6. Extracción de mucilago, en diversos tiempos de operación del equipo de burbujeo y diferentes relaciones ñame/agua para ñame espino y criollo. 


\section{CONCLUSIONES}

El proceso de extracción de almidón por burbujeo es dependiente del tiempo de duración del proceso como de la cantidad de agua, las cuales influyen el rendimiento en la extracción del mismo.

La obtención del mucilago aumenta a medida que el tiempo de operación se incrementa, mostrando ser influenciada además por la cantidad de agua presente en la pasta de ñame.

Mediante el uso del proceso continuo de burbujeo se obtienen mayores extracciones de almidón en la especie de ñame D. rotundata en comparación con D. alata.

\section{AGRADECIMIENTOS}

Los autores manifiestan agradecimientos, al programa jóvenes investigadores e innovadores de Colciencias, al igual que la Universidad De Sucre; por su entera colaboración en el desarrollo de las actividades involucradas en el desarrollo del mismo.

\section{REFERENCIAS}

Agronet, 2013. Sistema de información del agro colombiano.http://www.agronet. gov.co. [Octubre 13, 2013].

Alvis, A., Veléz, C. y Randa, M. 2008. Composición de ñames frescos cultivados en Colombia y sometidos a freído por inmersión. Información tecnológica 19(1): 3-10.

Anuario, A. 1994. Anuario a granja do ano. Sao Paulo, Centauros, p 123 - 243.
Aparecida, T., Pereira, J., Guerreiro, M., Pimienta, C., Velloso, M. y Pereira, L. 2011. Caracterização físicoquímica da mucilagem. Ciênc. agrotec 35(5):973-979.

Coursey, D. 1980. Yams-Dioscorea spp. (Dioscoreaceas). En: Simmnonds, N. Y. Evolution of crop plants, New York: Ed. Longmans., p. 70-74.

Corpoica, 2003. Concepción de un modelo de agroindustria rural para la elaboración de harina y almidón a partir de raíces y tubérculos promisorios, con énfasis en los casos de achira (Cannaedulis), arracacha (Arracaciaxanthorriza) y ñame (Dioscorea sp.) Informe Técnico Final. Corpoica; Pronata, Tibaitatá, Colombia. p 112.

Daiuto, E., Cereda, M., Sarmento, S. y Vilpoux, O. 2005. Effects of Extraction Methods on Yam (Dioscorea alata) Starch Characteristics. Starch - journal (57): 153-160.

FAOSTAT, 2011. FAO. http: / / faostat3. fao. org/ faostatgateway/ go/ to/ browse/ Q/ QC/ E. [Octubre 17, 2013].

Fu, Y., Chen, S., \& Lai, Y. 2004. Centrifugation and foam fractionation effect on mucilage recovery from Dioscorea (yam) tuber. Journal of Food Science, 69:509 - 514

Fu, Y., Huang, P. y Chu, C. 2005. Use of continuous bubble separation process for separating and recovering starch and mucilage from yam (Dioscorea pseudo 
japonica yamamoto). LWT (38):735-744.

\section{Hata, Y., Arteaga D., Buitrago, G., y Álaverez,}

A. 2003. Evaluación del contenido de sapogeninas en variedades nativas de ñame (Dioscorea spp.), provenientes de la colección de la Universidad de Córdoba. Col. Cienc. Quím. Farm. 32(2):149-157.

Misaki, A., Ito, T. y Harada, T. 1972. Constitutional Studies on the Mucilage of "Yamanoimo" Dioscorea batatas Decne, forma Tsukune: isolation and structure of a mannan. Agricultural and BiologicalChemistry (36):761-771.

Pérez, J. y Sayas, Y. 2010. Desarrollo de un proceso de burbujeo continúo para la extracción y recuperación de almidón y mucilago de ñame, Ingeniería agrícola, Universidad de sucre, Sincelejo, colombia

Reina, A. 2012. El cultivo de ñame en el Caribe Colombiano. Banco de la Republica, Cartagena, Colombia. p 34.
Salcedo, J., Montes, E., Zapata, J., Márquez, D. y Díaz, M. 2010. Obtención de jarabes de fructosa a partir de hidrolizados enzimáticos de almidón de ñame (Dioscorea alata y Dioscorea rotundata). VITAE 17(3):243-251

TSAJ, S; TAI, F. 1984. Studies on the mucilages from tuber of yam (Diocorea alata Linn). Isolation and purification of the mucilage. Journal of Chinese agriculturalchemical society (22):88-94.

Vidal, C. 2010. El ñame espino (Dioscorea rotundata Poir.): una opción en la producción de jarabes edulcorantes intermedios para la industria alimentaria. Revista de Investigación Agraria y Ambiental 1(2):19-28.

Yeh, A., Chan, T., y Chuang, G. (2008). Effect of water content and mucilage on physico-chemical characteristics of Yam (Discorea alata Purpurea) starch. Journal of Food Engineering, 106-114. 